\title{
The influence of sub-100nm scattering on
}

\section{High-Energy Electron Beam Lithography}

Erik H. Anderson, Deirdre L. Olynick, Weilun Chao, Bruce Harteneck, and Eugene Veklerov

E.O. Lawrence Berkeley National Laboratory, Berkeley CA 94720

\begin{abstract}
Electron beam lithography tools have evolved in the direction of higher beam energy in order to achieve high-resolution, fine feature definition. As the beam energy is increased, the "forward" scattering is reduced and the "backscatter" range is increased. Over the years, tools became available at $20 \mathrm{KV}$ then $50 \mathrm{KV}$ and now $100 \mathrm{KV}$ operation is common. Operation at higher voltages has several advantages, such as better resolution and process latitude due to reduced forward scattering, and a few disadvantages such as higher dose requirements, substrate heating, and lower contrast for back scatter electron alignment and calibration signals (due to reduced primary electron backscattering generation in thin flim). The backscatter range for $100 \mathrm{KV}$ on silicon is about $27 \mu \mathrm{m}$ compared to $8 \mu \mathrm{m}$ at $50 \mathrm{KV}$ resulting in different strategies for efficient proximity correction. However, even at $100 \mathrm{KV}$, scattering in an intermediate range is observed and must be taken into account in order to achieve good line-width control at the highest resolution. Measurements of the scattering range for both $50 \mathrm{KV}$ and $100 \mathrm{KV}$ have been made using the point exposure distribution measurement technique. ${ }^{[1]}$ For comparison, measurements taken on the same wafer at different voltages, show that $50 \mathrm{KV}$ and $100 \mathrm{KV}$ scattering range functions overlap, after normalizing for the different resist
\end{abstract}


sensitivity, at length scales below $0.5 \mu \mathrm{m}$, suggesting a common mechanism, which is independent of the initial electron energy. For thin resists, this suggests that the significant resolution difference between $50 \mathrm{KV}$ and $100 \mathrm{KV}$ lithography is limited to the "forward" scattering effect as the incident electrons traverse the resist. Extrapolating the scattering function to the approximate beam diameter of $10 \mathrm{~nm}$ allows an impulse response function to be numerically determined. The convolution of this function gives reasonably good agreement with dose versus line-width measurements.

\section{Introduction}

In electron beam lithography, the incident beam is scattered by the resist and the substrate resulting in unwanted dose being deposited in areas outside of the desired exposure region. Normally, this scattering is discussed in terms of "backscatter" which is the result of a large number of electron scattering events and where resist can be exposed quite some distance from the electron beam position and "forward" scattering which is the result of a small number of scattering events as the electron beam passes through the resist. In thicker resist, forward scattering will have a larger range than thin resist. For high accelerating voltage systems, forward scattering has a significantly smaller range than backscatter. In reality, scattering is not so neatly divided into two distinct regimes. A practical technique for investigating scattering is the point exposure distribution measurement technique as described by Rishton and Kern. ${ }^{[1]}$ In this technique, a series of dot point exposures with exponentially increasing dose over many orders of magnitude is carried out using a high-contrast resist as the scattering detector. 
Figure 1 shows a set of dots with three different exposure doses and an ideal infinite-contrast resist which results in dots whose diameters map out the intersection of the "cut-off" dose and the applied dose profile. With real resist materials, the contrast is not infinite and the resulting dots will have a less than ideal development with some ambiguity as to the exact diameter. Never the less, SEM inspection of dots in real resist yields valuable diameter versus dose data. Analysis of this data gives insight into the scattering function profile which can be compared with other lithographic data such as line-width versus dose measurements.

\section{Experimental Details}

The tool used is a modified Leica Microsystems VB6-HR ${ }^{[2]}$ system coupled with a unique digital pattern generator (DPG), and control software developed internally at Berkeley Laboratory ${ }^{[3]}$. The column consists of a Thermal Field Emission source for high-brightness giving small probe size and high current. The accelerating voltage is variable between $20 \mathrm{KV}$ and $100 \mathrm{KV}$ with measurements taken at $50 \mathrm{KV}$ and $100 \mathrm{KV}$. The stage has travel over approximately $150 \mathrm{~mm}$ by $150 \mathrm{~mm}$ area and incorporates a 3 axis $(\mathrm{x}, \mathrm{y}, \theta), \lambda / 1024(0.6 \mathrm{~nm})$ resolution interferometer for positional measurement, feedback, and control. The beam diameter is estimated to be about $10 \mathrm{~nm}$ at the operating conditions used. The resist used is hydrogen silsesquioxane, (HSQ) described by Falco et. A1. ${ }^{[4]}$ This resist has high resolution, modest dose requirements, and is commercially available at high purity. The HSQ solution used is FOx-15 from Dow-Corning and diluted with methyl isobutyl ketone (MIBK) so that final resist thickness is about 40nm 
after spin coating. The resist is soft baked at $170 \mathrm{C}$ for 30 minutes. After electron beam exposure it is developed in $0.26 \mathrm{~N}$ tetramethyl ammonium hydroxide (TMAH), (Shipley corporation LDD26-w) for 1 minute. A computer program prepares the data sets with a series of dot shapes increasing in dose in a geometric progression. The data sets cover a large range of 9 orders of magnitude so that short range, intermediate range, and long range scattering is observed on the same wafer. Figure 2 shows low voltage SEM micrographs of actual exposed dots. Figure 3 shows a plot of dot radius data taken on the same wafer at $50 \mathrm{KV}$ and $100 \mathrm{KV}$ on silicon. The $100 \mathrm{KV}$ exposure was done first and then the voltage was reduced. The wafer remained in the system while the high voltage was changed and the system realigned. The wafer was then exposed with the dot pattern again at $50 \mathrm{KV}$ and developed. Therefore any differences due to development conditions of temperature or time were eliminated. In-vaccum delay sensitivity of HSQ over short times has not been observed. Because the sensitivity at 50KV differs from $100 \mathrm{KV}$ by a factor of 2 , the $50 \mathrm{KV}$ data is plotted versus twice the actual dose, normalizing the data for the resist sensitivity. Figure 4 shows dot data on a $\mathrm{Cr}(5 \mathrm{~nm}) / \mathrm{Au}(12 \mathrm{~nm})$ plating base on silicon, a hard baked $180 \mathrm{~nm}$ thick polymer layer on silicon, and a bare silicon substrate. Figure 4 suggests that the plating base and polymer materials do not significantly change the form of the scattering function. The amount of scatter is different between the different substrates and lithography on the polymer is generally higher resolution. The area around the dots of the plating base wafer is electroplated with nickel to improve SEM contrast and mirror the conditions in which diffractive x-ray optics are fabricated. This data 
on the plating base wafer extends well into the $100 \mathrm{KV}$ backscatter range and the traditional gaussian functional form is observed when re-plotting this data in the form of $\log ($ dose $)$ versus radius ${ }^{2}$. Figure 5 shows the $100 \mathrm{KV}$ dot data re-plotted to highlight the gaussian functional form which will be a straight line since:

$\log ($ Dose $)-(r / \beta)^{2}=C$. The slope of the straight line gives the backscatter range of $\beta=26 \mu \mathrm{m}$. The data for $50 \mathrm{KV}$ is plotted in figure 6 to highlight the backscatter range with a derived range, $\beta=8 \mu \mathrm{m}$.

\section{Fitting the measured data}

The dot size versus dose data is well described with a gaussian function for the long range scattering as seen by the straight line segments of figures 5 and 6 . For analytical convenience, a sum of gaussian terms is a desirable fit of the scattering data. From the mathematics of the central limit theorem we would expect gaussian distributions from large numbers of independent scattering events even if each individual scattering event has a non-gaussian distribution. However, the data is not well approximated by a sum of gaussian functions. By visual inspection, the $\log (\mathbf{d o s e})$ is linear in $\log (\mathbf{r})$ over a range of values and so an expression of the form, $\log (\operatorname{dose})=\mathbf{a} 0+\mathbf{a} 1 \log (\mathbf{r})+\mathbf{a} 2(\log (\mathbf{r}))^{2}+\mathbf{a 3}(\log (\mathbf{r}))^{3}+\mathbf{a} \mathbf{4}^{2}$ was used. Note that the last term, $\mathbf{a} 4 \mathbf{r}^{2}$ dominates the expression for large values of $\mathbf{r}$ and so this approximation will yield a gaussian functional form as expected from physical considerations. Figure 7 shows the data and fitting function for $50 \mathrm{KV}$ data while figure 4 shows the $100 \mathrm{KV}$ data. The coefficients are determined using the linear algebra method of singular value decomposition. The first coefficient an, 
represents the scale and is determined by the units in use. The fifth coefficient, a4, represents the backscatter effect and is related to the backscatter range by $\mathbf{a 4}=$ $1 / \boldsymbol{\beta}^{2}$. The coefficient a1 represents a power law relationship between the scattering range and the dose. Concentrating on the linear sections of the shortrange scattering we find the slope to give a power law value of approximately 2.16 for $50 \mathrm{KV}$ and a value of 2.12 for $100 \mathrm{KV}$. These values are remarkably close suggesting a common physical scattering mechanism.

\section{Impulse Response and comparison with Linewidth versus Dose data}

We have investigated if the point exposure distribution measurement technique can be used to predict linewidth and process latitude. First the dot data is fitted to an analytical expression using the above formula and then extrapolated. Here the $100 \mathrm{KV}$ dots on $\mathrm{Cr} / \mathrm{Au}$ plating base was used. Unfortunately, the scattering function for small ranges is divergent so the extrapolation cannot continue to zero. A cutoff is chosen based on the finite beam width of about $10 \mathrm{~nm}$, or $5 \mathrm{~nm}$ in radius. The extrapolated two-dimensional function is then summed up to give an equivalent one-dimension impulse function. Using this impulse response we calculate the dose versus linewidth for a periodic set of lines on $\mathrm{Cr} / \mathrm{Au}$ plating base

at $100 \mathrm{~nm}, 80 \mathrm{~nm}$ and $60 \mathrm{~nm}$ as shown in figures 8,9 , and 10 . These lines were measured after a small amount of nickel material was plated to help with the SEM imaging. In these figures the only non-experimentally determined model parameter is the cutoff limit of the scattering function. Agreement between experiment and model is generally good considering that the model of the development process is highly simplified to approximate an infinite contrast resist. 


\section{Conclusion}

The point exposure distribution measurement technique has been used to measure scattering in the short, intermediate, and long (backscattering) range regimen for electron energies of $50 \mathrm{KV}$ and $100 \mathrm{KV}$ on silicon and silicon with plating base substrates. In the long-range or backscatter regimen, the dot data gives good agreement with a gaussian functional form with $\beta=26 \mu \mathrm{m}$ and $\beta=8 \mu \mathrm{m}$ for $100 \mathrm{KV}$ and $50 \mathrm{KV}$ respectively. In the short and intermediate range, below $0.5 \mu \mathrm{m}$, the data for $50 \mathrm{KV}$ and $100 \mathrm{KV}$ overlap when plotted with normalized sensitivity. This suggests that the mechanism of scattering and resist exposure is independent of the initial electron energy. It also suggests that a reduction in the forward scattering of the primary beam is the most significant difference between $100 \mathrm{KV}$ and $50 \mathrm{KV}$ operation. The scattering data can be well fitted using a linear combination of $\log (\mathbf{r}), \log (\mathbf{r})^{2}, \log (\mathbf{r})^{3}$ and $\mathbf{r}^{2}$ functions. Extrapolating this function to a cutoff length gives an impulse function, which provides reasonable agreement with linewidth versus dose data for $100 \mathrm{~nm}, 80 \mathrm{~nm}$, and $60 \mathrm{~nm}$ period lines.

\section{Acknowledgements}

This work was supported by the Defense Advanced Research Project Agency Advanced Lithography Program through the U.S. Department of Energy under Contract No. DE-AC03-76SF00098. 


\section{References}

[1] S.A. Rishton and D.P. Kern, "Point exposure distribution measurements for proximity correction in electron beam lithography on a sub-100nm scale,” J. Vac. Sci. Technol. B 5 (1) Jan/Feb 1987

[2] B.H. Koek, T. Chisholm, A.J.V. Run, J. Romijn, and J.P., Davey, “An electron beam lithography tool with a Schottky emitter for wide range applications," Microelectronic Engineering 23, 81, (1994).

[3] E.H. Anderson, V. Boegli, and L.P. Muray, "Electron Beam Lithography Digital Pattern Generator and Electronics for Generalized Curvilinear Structures, ”J. Vac. Sci. Technol. B 13, 2529 (1995).

[4] Falco C.M.J.M. van Delft, Jos P. Weterings, Anja K. van LangenSuurling, and Hans Romijn, "Hydrogen silsesquioxane/novolak bilayer resist for high aspect ratio nanoscale electron-beam lithography," J. Vac. Sci. Technol. B 18(6) Nov/Dec 2000, pp 3419-3423. 


\section{List of Figures}

Figure 1. The point exposure distribution measurement technique uses a high contrast resist as a spatial critical dose detector. The dot size as a function of dot dose can be used to trace out the scattering function. Each dot is separated by $200 \mu \mathrm{m}$ so that there is no interaction between the dots.

Figure 2. HSQ dots are observed by low voltage scanning electron microscopy. Each dot is measured using the built in linewidth annotation features of the SEM.

Figure 3. Dot data for HSQ resist on a silicon wafer at $50 \mathrm{KV}$ and $100 \mathrm{KV}$ operating conditions. The same wafer was used for this experiment to eliminate any differences due to development conditions. The $50 \mathrm{KV}$ dose data is multiplied by two in order to normalize it with respect to the $100 \mathrm{KV}$ sensitivity. The overlap of the data at length scales below $0.5 \mu \mathrm{m}$ suggests that the scattering and exposure mechanism is independent of the initial electron beam energy within some large range. The base dose, $\mathrm{D}_{0}$, was $3.2 \times 10^{-17}$ coulombs

Figure 4. A plot of a $100 \mathrm{KV}$ dot data set taken on a silicon wafer with $\mathrm{Cr} / \mathrm{Au}$ plating base. The solid line shows the fitting function. The base dose, $\mathrm{D}_{0}$, was $3.2 \times 10^{-17}$ coulombs 
Figure 5. Re-plotting the $100 \mathrm{KV}$ dot data of figure 4 as a function of radius squared shows the longer-range or backscattering functional form. A gaussian function when plotted in this fashion will show a straight line dependence. From the slope of the straight section the backscatter range of $26 \mu \mathrm{m}$ is determined. The base dose, $\mathrm{D}_{0}$, was $3.2 \times 10^{-17}$ coulombs

Figure 6. Re-plotting the $50 \mathrm{KV}$ dot data of figure 3 as a function of radius squared shows the backscattering functional form. A gaussian function when plotted in this fashion will show a straight line dependence. From the slope of the straight section the $50 \mathrm{KV}$ backscatter range of $8 \mu \mathrm{m}$ is determined. The base dose, $\mathrm{D}_{0}$, was $3.2 \times 10^{-17}$ coulombs

Figure 7. This shows the dot data and the fitted function for $50 \mathrm{KV}$ dots on a silicon substrate. The base dose, $\mathrm{D}_{0}$, was $3.2 \times 10^{-17}$ coulombs

Figure 8. Measured (dots) and calculated (solid) linewidth versus dose for $100 \mathrm{~nm}$ period lines.

Figure 9. Measured (dots) and calculated (solid) linewidth versus dose for $80 \mathrm{~nm}$ period lines. 
Figure 10. Measured (dots) and calculated (solid) linewidth versus dose for $60 \mathrm{~nm}$ period lines. 

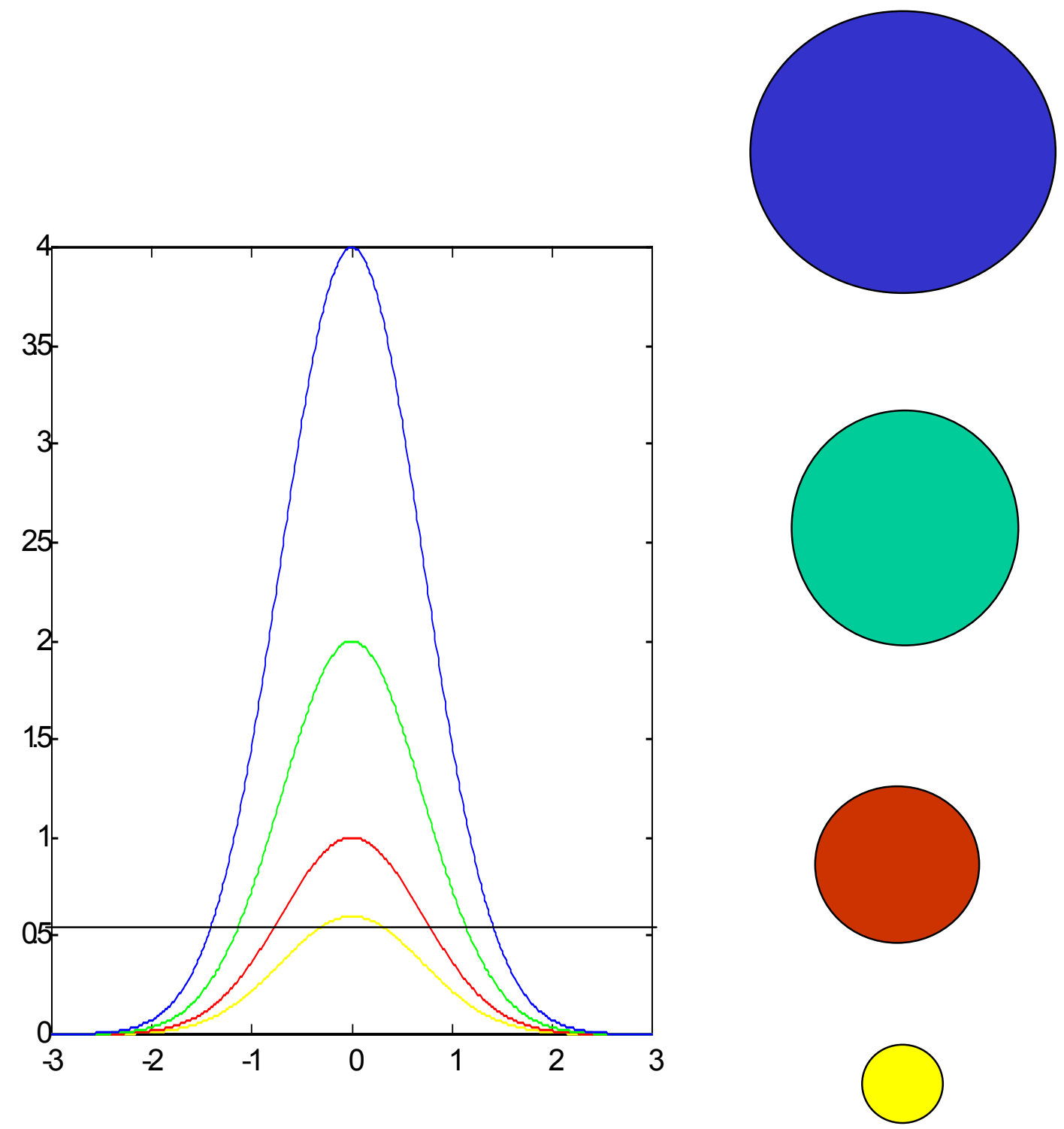

Figure 1. 

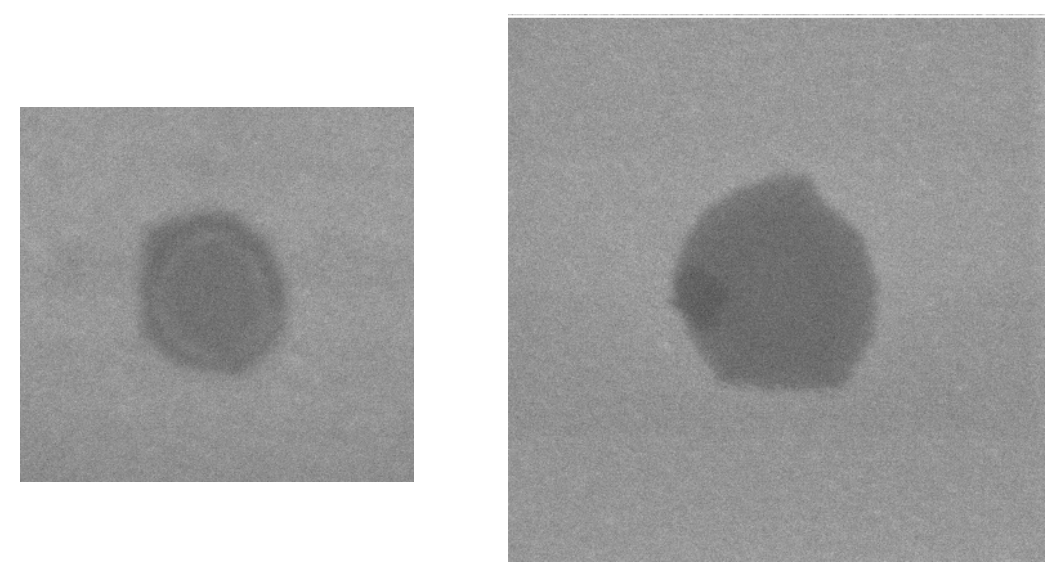

$\leftrightarrow \quad \leftrightarrow$

$300 \mathrm{~nm}$

$0.136 * 10^{8}$

$1.66 * 10^{8}$

$7.73 * 10^{8}$

\section{Increasing Dose}

Figrue 2 
$100 \mathrm{KV}$
$1 \mathrm{xDose} / \mathrm{D}_{0}$

$50 \mathrm{KV}$
$2 \mathrm{xDose}^{-\mathrm{D}_{0}}$

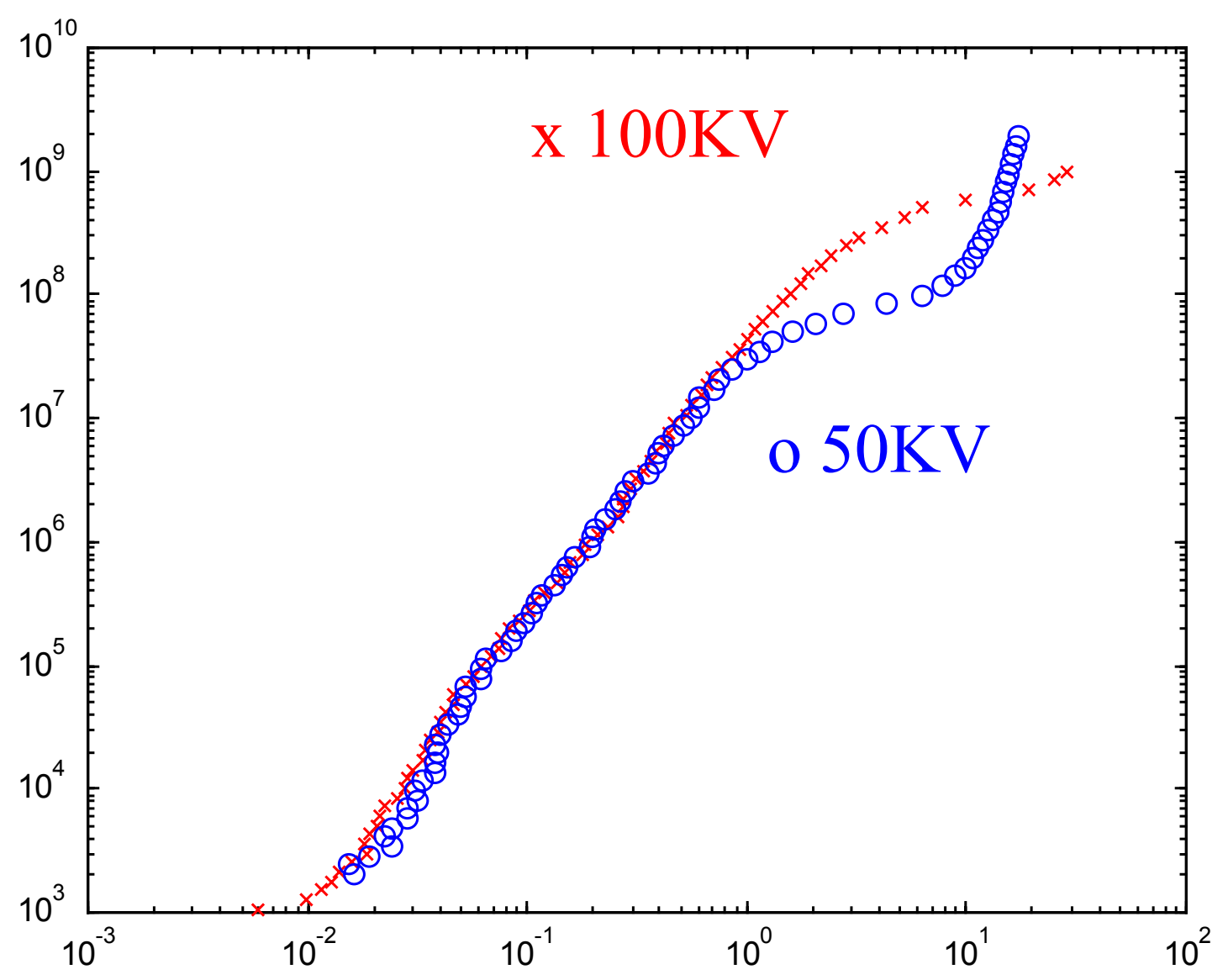

Dot Radius $[\mu \mathrm{m}]$

Figure 3 
$\underline{\text { Dose }}$ $\mathrm{D}_{0}$

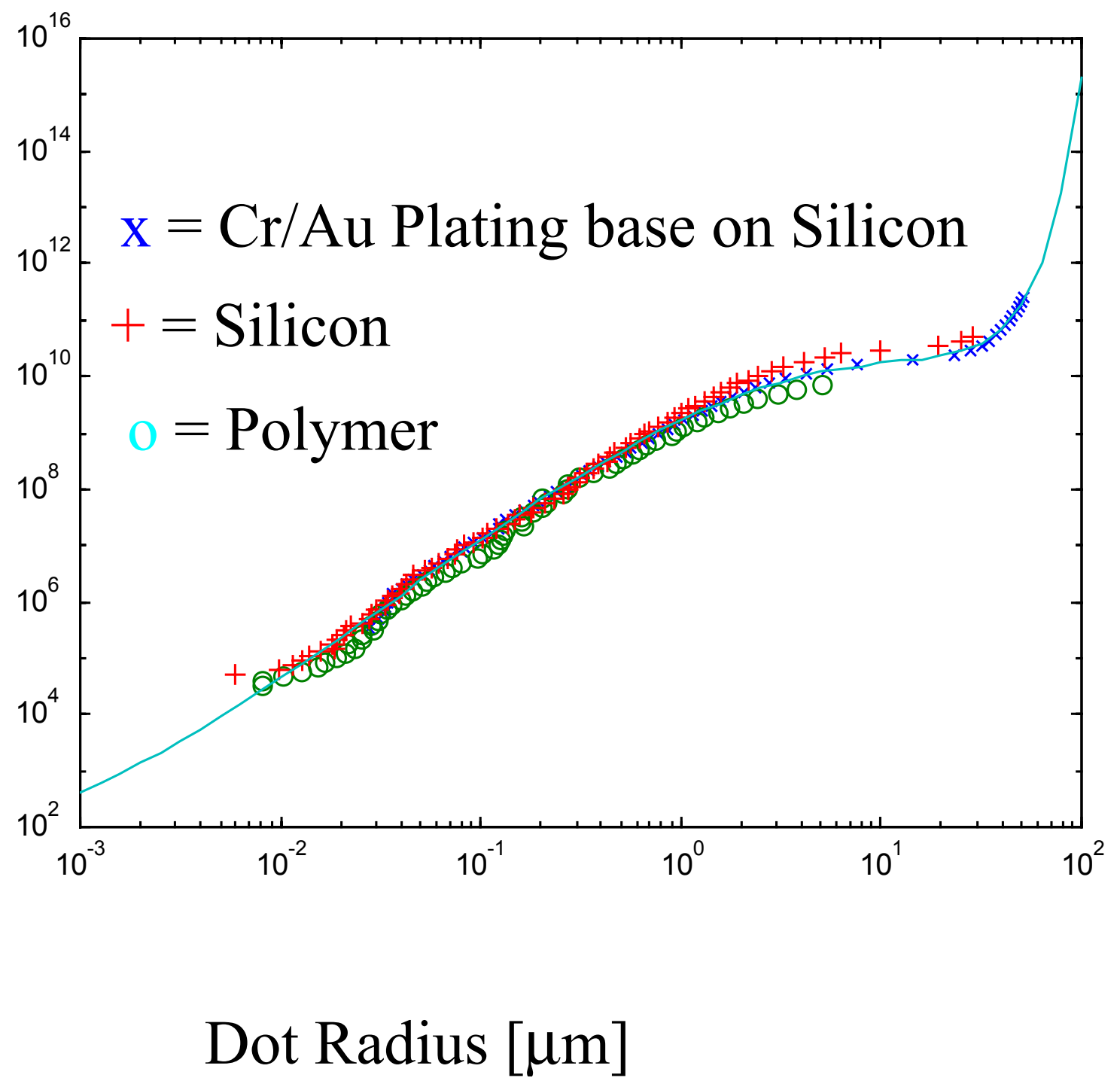

Figure 4 


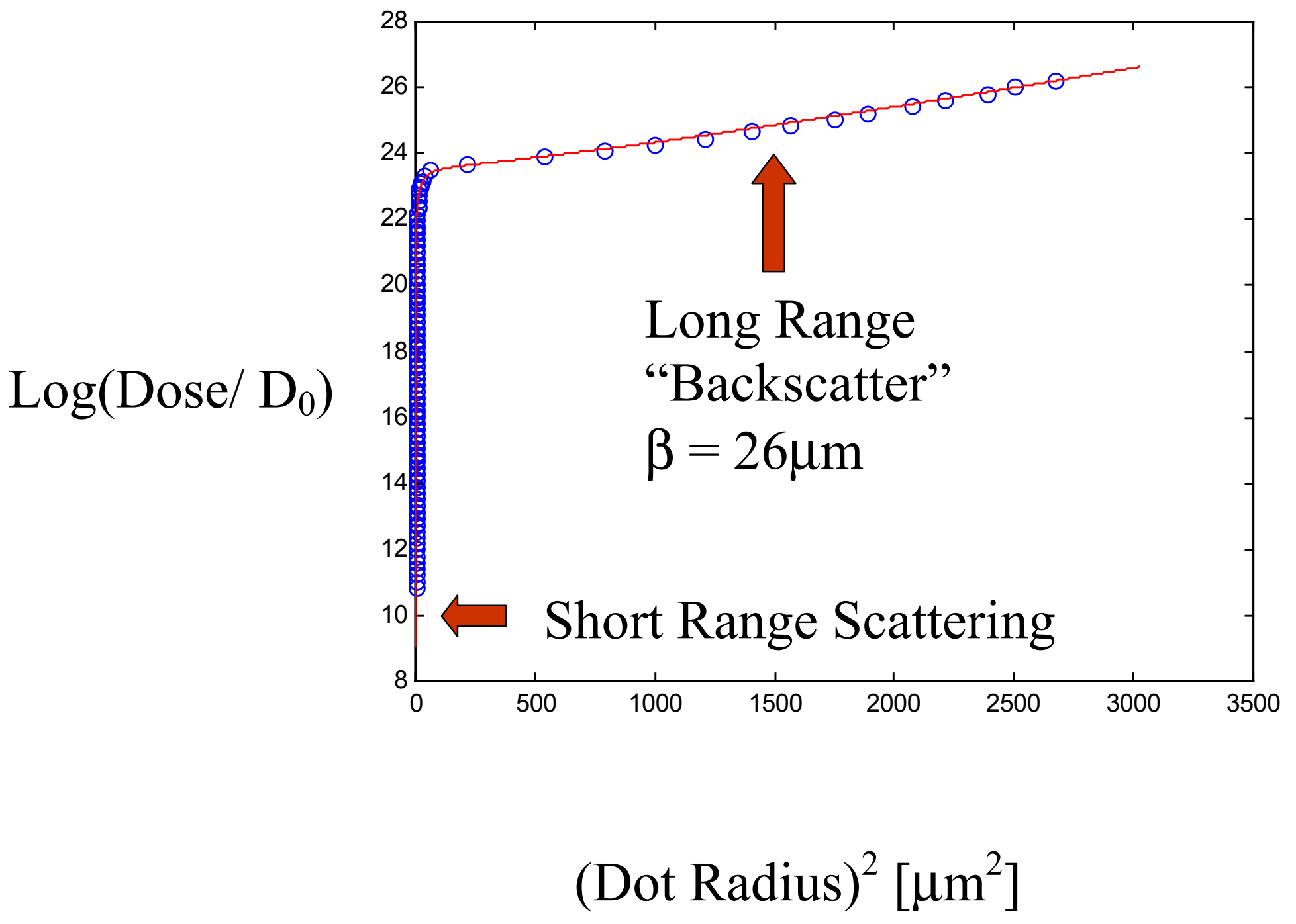

Figure 5 
Log (Dose/ $\left.\mathrm{D}_{0}\right)$

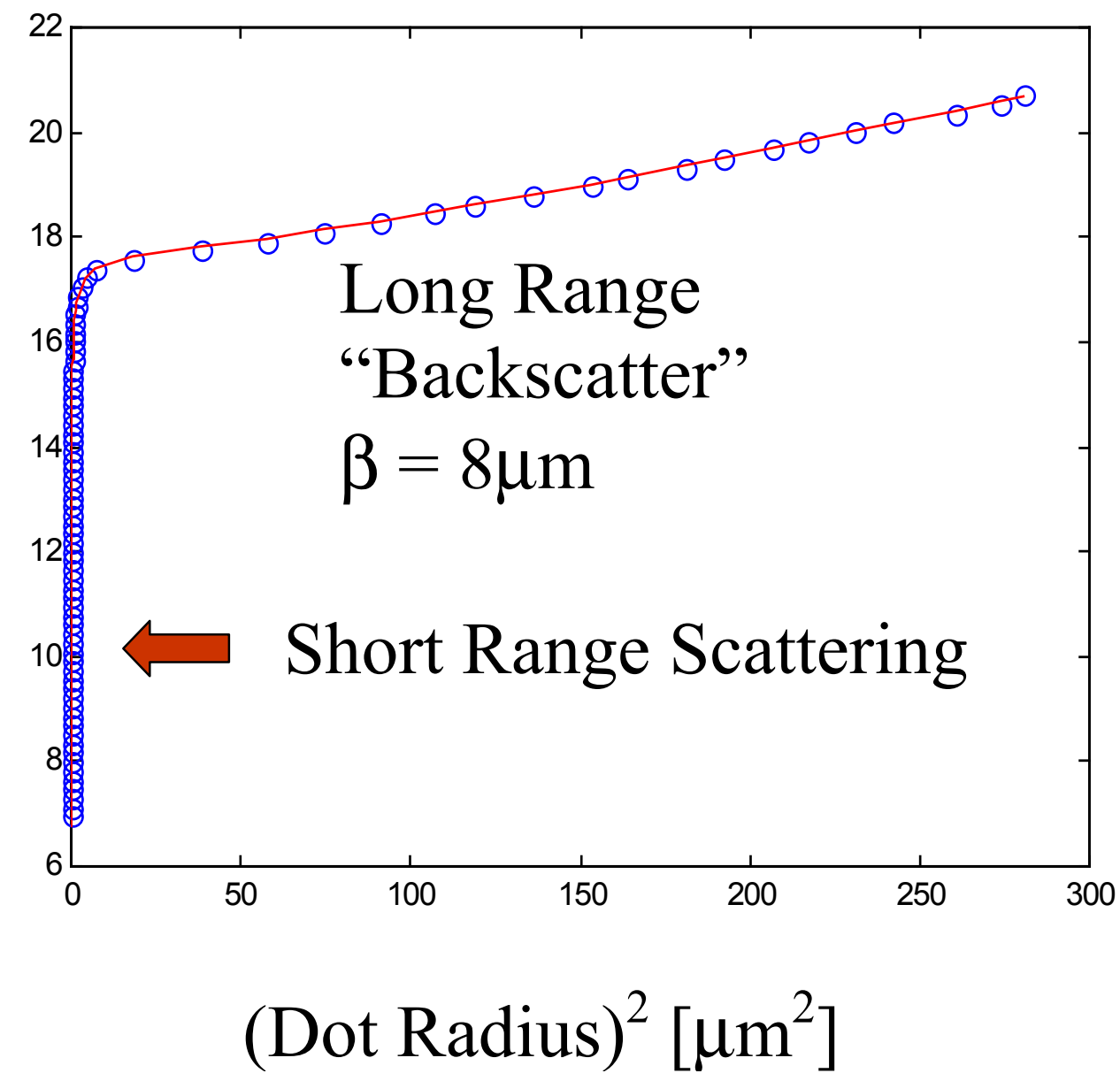

Figure 6 


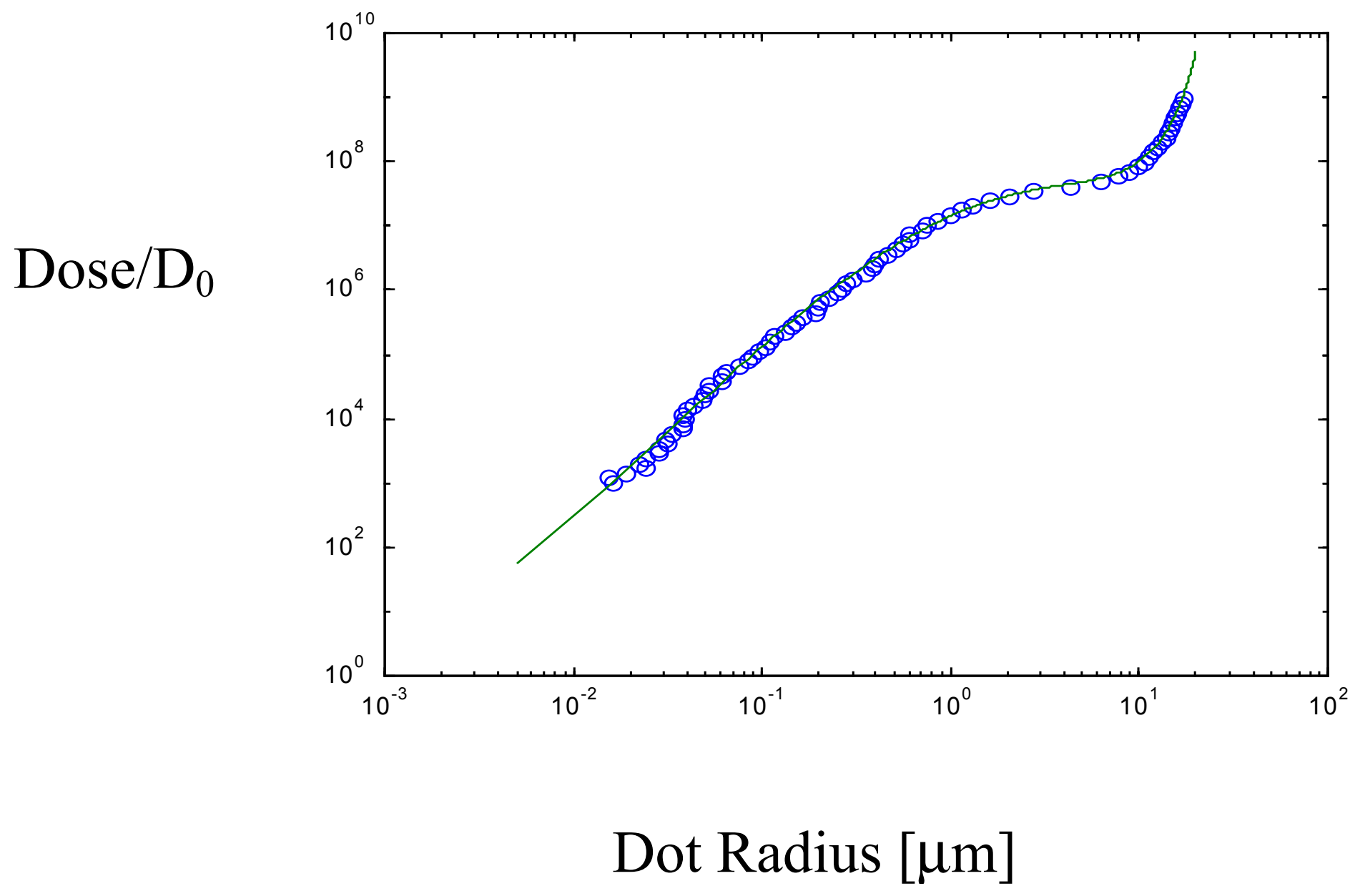

Figure 7 


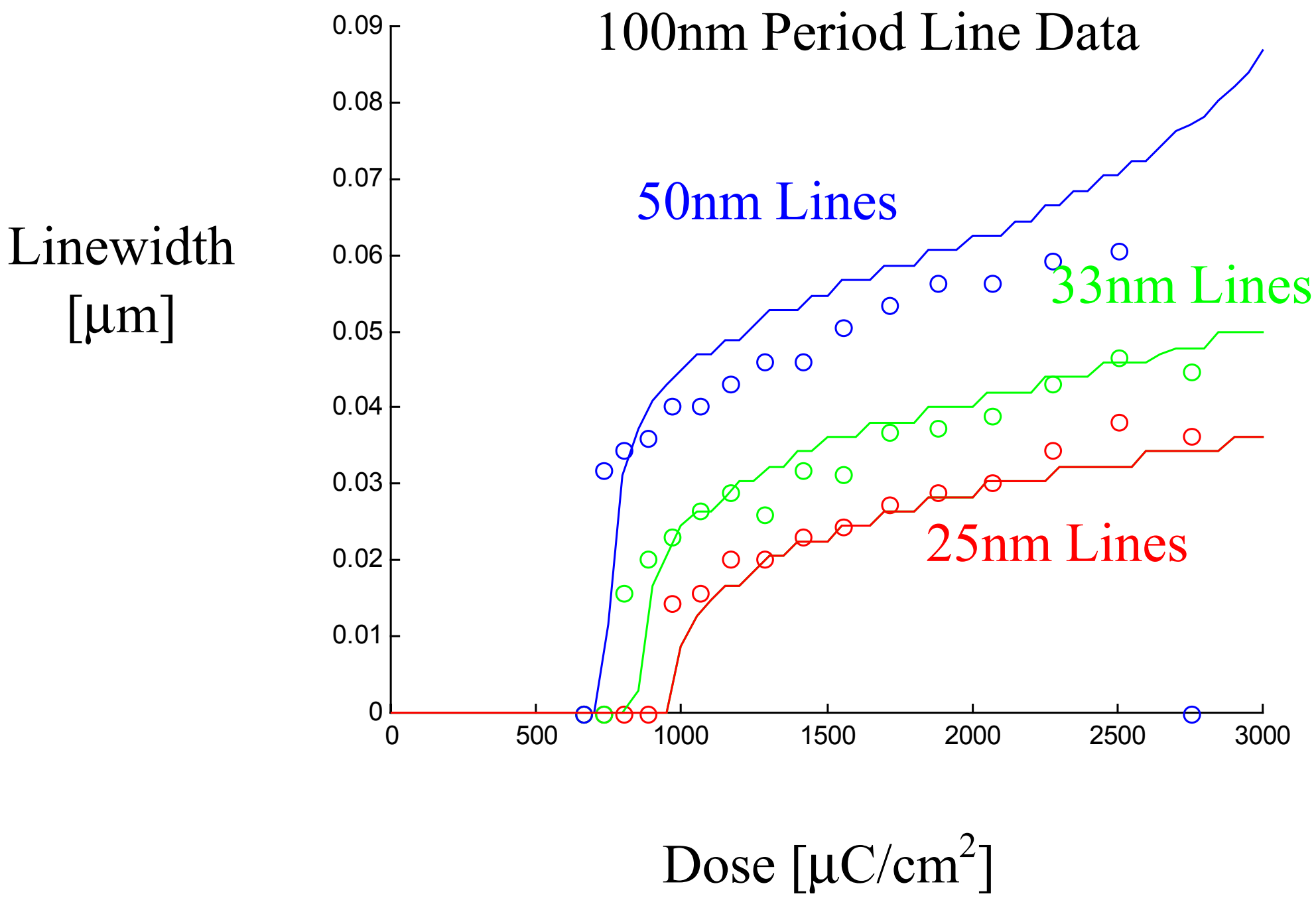

Figure 8 


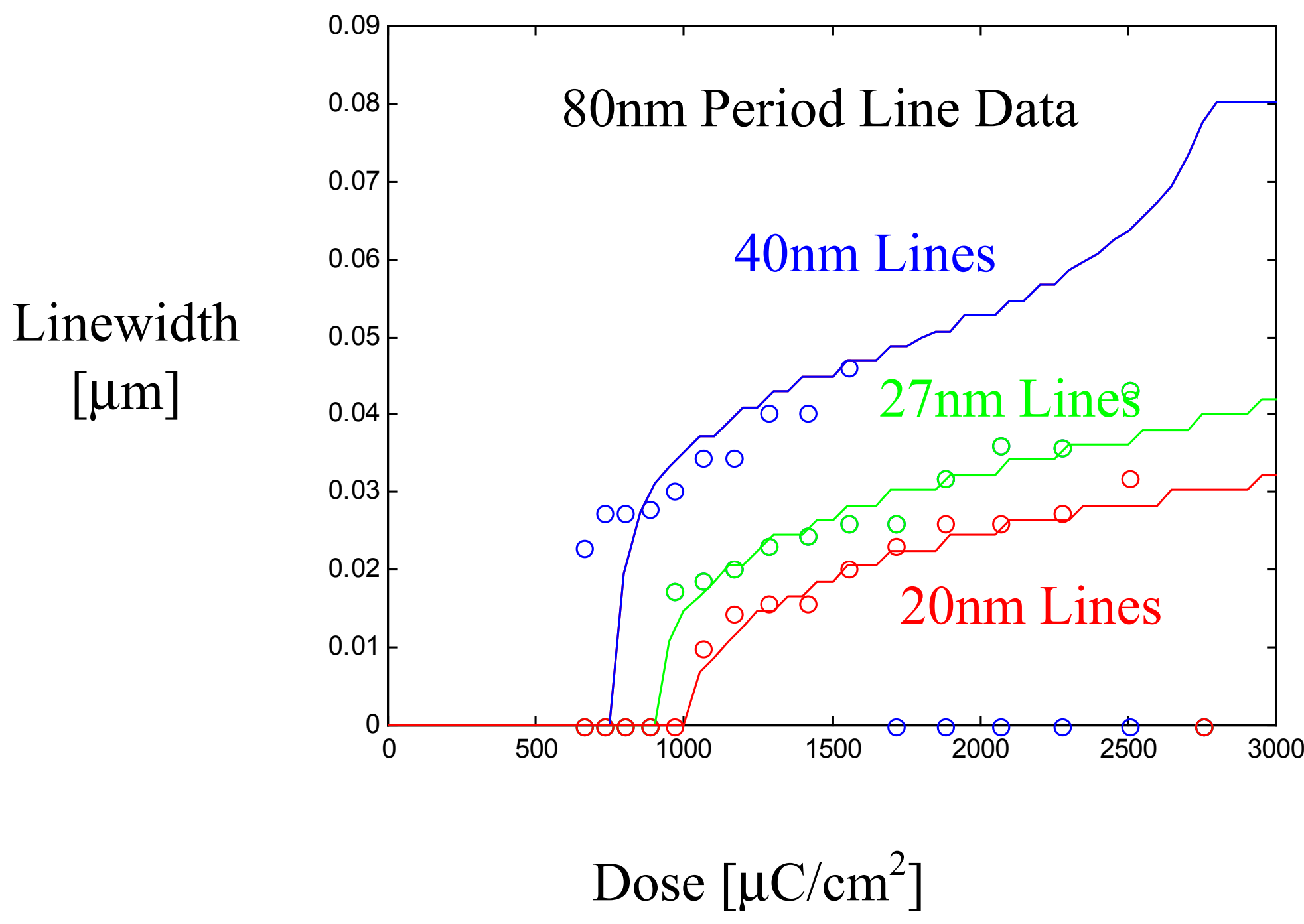

Figure 9 
Linewidth

$[\mu \mathrm{m}]$

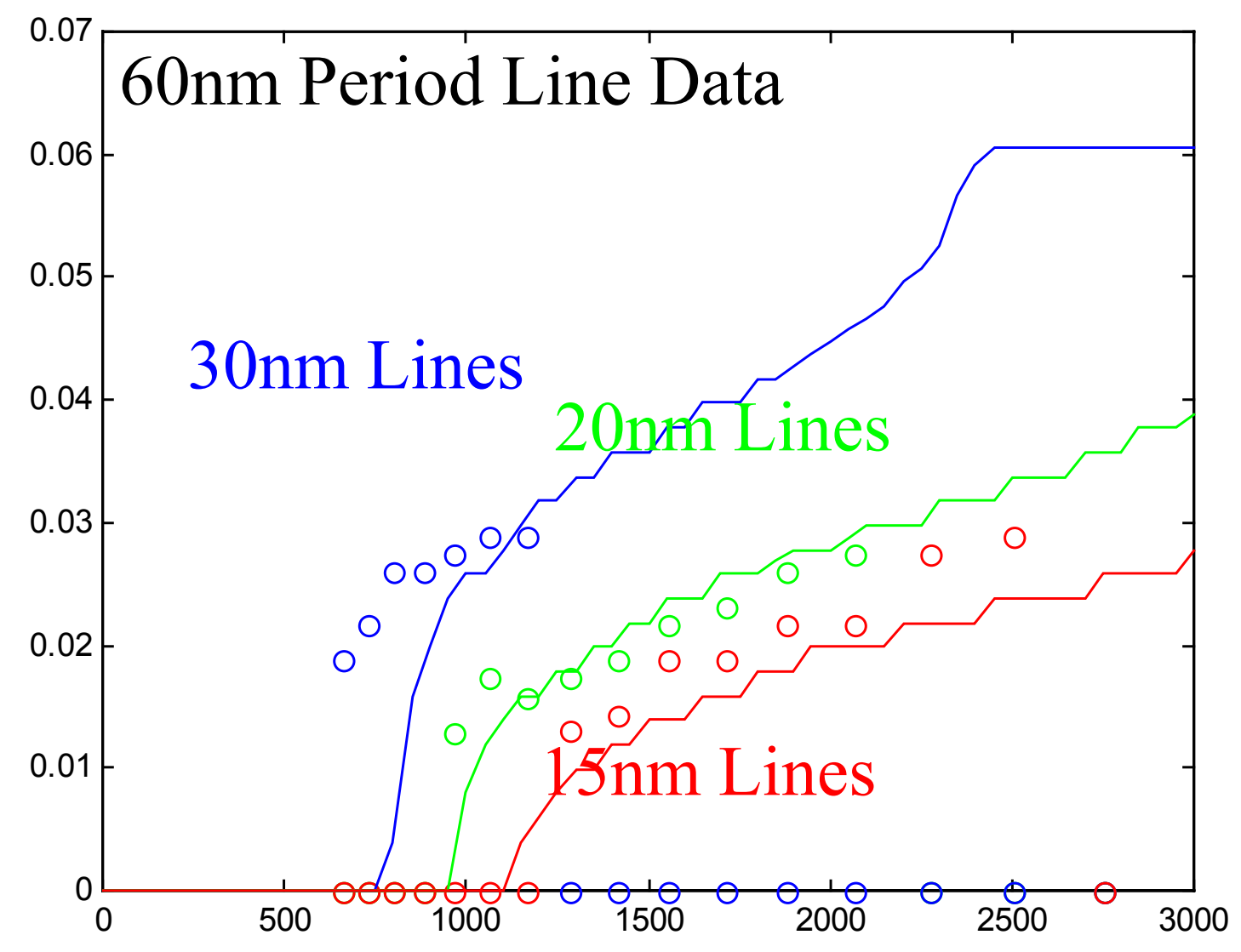

Dose $\left[\mu \mathrm{C} / \mathrm{cm}^{2}\right]$

Figure 10 